\title{
Clinical and Pathological Characteristics of Patients with High-Risk Breast Cancer Based on BRCA Mutation Profiles: A Retrospective Study
}

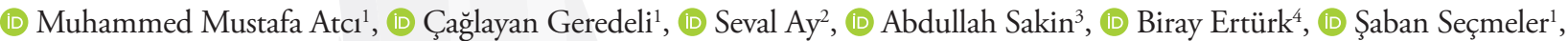
(1) Serdar Arıc1 ${ }^{1}$, (1) Ruhper Çekin ${ }^{1}$, (1) Nurgül Yaşar ${ }^{1}$, (1) Orçun Can ${ }^{1}$, (1) Şener Cihan ${ }^{1}$, (1) Mahmut Gümüşs

${ }^{1}$ Clinic of Medical Oncology, İstanbul Professor Doctor Cemil Tasçıŏlu City Hospital, İstanbul, Turkey

${ }^{2}$ Department of Medical Oncology, Medeniyet University, Göztepe Training and Research Hospital, İstanbul, Turkey

${ }^{3}$ Clinic of Medical Oncology, Van Training and Research Hospital, Van, Turkey

${ }^{4}$ Clinic of Medical Genetics İstanbul Professor Doctor Cemil Tasçıŏlu City Hospital, İstanbul, Turkey

\section{ABSTRACT}

Objective: This study aimed to determine the differences in clinicopathological features of Turkish patients with high-risk breast cancer based on the mutation status of two breast cancer susceptibility genes (BRCA1/2).

Materials and Methods: This study enrolled patients with invasive breast cancer who have been evaluated for BRCA1/2 mutations due to the presence of high-risk factors admitted to two tertiary referral centers in Turkey. Clinical and histopathological features were analyzed in BRCA1 mutation carriers, BRCA2 mutation carriers, and non-carriers.

Results: A total of 302 patients with a mean age of $44.2 \pm 9.9$ (22-82) years were included. BRCA1/2 mutation was found in 75 (24\%) patients, of whom $41(13.6 \%)$ were BRCA1 mutation carriers and $37(12.3 \%)$ were BRCA2 mutation carriers. Moreover, $104(34.4 \%)$ and $4(1.3 \%)$ patients had family history of breast and ovarian carcinoma, respectively. The rates of triple negativity (56.1\%), histologic grade $3(65.9 \%)$, and lymphovascular invasion (78\%) were significantly higher in BRCA1 mutation carriers than in non-carriers and BRCA2 mutation carriers. Furthermore, $87 \%$ of triple-negative BRCA1 mutation carriers had histologic grade 3 tumors compared with $38.9 \%$ in non-triple-negative BRCA1 mutation carriers, and the difference was significant. Conclusion: Findings of this study showed that BRCA1-related breast cancers represent a distinct group with unique pathological features, which are usually associated with a poor prognosis.

Keywords: BRCA, breast cancer, Triple-negative, lymphovascular invasion, grade

Cite this article as: Atcı MM, Geredeli Ç, Ay S, Sakin A, Ertürk B, Seçmeler S, Arııı S, Çekin R, Yaşar N, Can O, Cihan Ş, Gümüş M. Clinical and Pathological Characteristics of Patients with High-Risk Breast Cancer Based on BRCA Mutation Profiles: A Retrospective Study.

Eur J Breast Health 2021; 17(2): 123-127

\section{Key Points}

- The rates of triple negativity, histologic grade 3 , and lymphovascular invasion were significantly higher in BRCA1 mutation carriers.

- While most of the triple-negative BRCA1 mutation carriers had histologic grade 3 tumor, it was not common in non-triple-negative BRCA1 mutation carriers.

- These findings showed that BRCA1-related breast cancers have pathological features related with poor prognosis.

\section{Introduction}

Evaluation of two breast cancer susceptibility genes, namely, BRCA1 and BRCA2, is essential in patients with a predisposition to carry these mutations. Number of family members with breast cancer, young age at diagnosis, bilateral disease, and family history of ovarian cancer have been proposed as predictors of BRCA 1/2 mutations in patients with breast cancer (1). The frequency of BRCA1 and BRCA2 mutations may vary between ethnic groups (2). However, only a few studies have investigated the frequency of BRCA1/2 mutations in Turkish patients with breast cancer $(3,4)$. Additionally, it is crucial to determine the clinical characteristics and pathological features in BRCA1/2 mutation carriers, which is also essential to define the differences between them and BRCA mutation non-carriers (5). In this study, we aimed to elucidate the frequency of BRCA1/2 mutations in a large series of patients with high-risk breast cancer and its relationship with personal/family history 
profiles and to perform clinicopathological analysis of patients with BRCA1/2-associated breast carcinoma from two tertiary referral centers in Turkey.

\section{Materials and Methods}

Among patients diagnosed with invasive breast cancer between 2015 and 2020 in two tertiary referral centers in İstanbul, patients with breast cancer who have been evaluated for BRCA1/2 mutations due to the presence of high-risk factors including younger age at diagnosis (<40 years old), male sex, bilateral localization of the tumor, and personal/family history of breast and ovarian cancer were enrolled in this study. BRCA1/2 mutations were investigated using next-generation sequencing. Patients' data including demographic information and frequency of BRCA mutation according to personal/ family history risk factors were retrospectively analyzed. In addition, BRCA1 mutation carriers, BRCA2 mutation carriers, and non-carriers were analyzed in terms of the clinical and histopathological features including hormonal status, histologic grade, lymphovascular invasion, and perineural invasion.

The study was conducted in accordance with the Declaration of Helsinki and approved by the Ethics Review Committee of İstanbul Professor Doctor Cemil Tasçıŏlu City Hospital (approval no. 48670771-514.10/210).

\section{Statistical analysis}

Data were analyzed using SPSS 22.0 software program. All continuous values were presented as median (range) and mean \pm standard deviation. Categorical data were expressed as a percentage and number. Associations between patients' BRCA mutation status and demographical, clinical, and histopathological characteristics were assessed using the chi-square test. The $\mathrm{p}$-value of $<0.05$ was considered significant.

\section{Results}

A total of 302 patients with a mean age of $44.2 \pm 9.9$ (22-82) years, of whom five were male, were included in this study. Moreover, 203 (68.4\%) and 94 (31.6\%) female patients had premenopausal and postmenopausal states, respectively. Breast carcinoma was localized bilaterally in $21(7 \%)$ patients, and only $6 \%$ of our patients had metastatic disease. A total of $75(24 \%)$ patients were BRCA1/2 mutation carriers. Forty-one (13.6\%) patients were BRCA1 mutation carriers, 37 (12.3\%) were BRCA2 mutation carriers, and three (1\%) were both BRCA1 and BRCA2 mutation carriers. First-I second-degree relatives of $104(34.4 \%)$ and 4 (1.3\%) patients had history of breast and ovarian carcinoma, respectively. Additionally, the frequency of BRCA mutation was the highest in patients with breast carcinoma with a family history of ovarian carcinoma (75\%), followed by patients with breast carcinoma with a personal history of ovarian carcinoma $(62.5 \%)$, and male patients with breast cancer $(60 \%)$.

Comparison of demographic, clinical, and pathological data of patients according to the BRCA mutation profile are shown in Table 1. Most of the BRCA1 mutation carriers $(75.6 \%)$ were $>40$ years old $(\mathrm{p}<0.05)$. Among BRCA1 mutation carriers, 19.5\%, 43.9\%, and 31.7\% were 30-39, 40-49, and 50-59 years of age, respectively. Among BRCA2 mutation carriers, $43.2 \%, 31.7 \%$, and $5.4 \%$ were $30-39$, 40-49, found among BRCA1 mutation carriers, BRCA2 mutation carriers, and BRCA non-carriers in terms of menopausal status and body mass index (Table 1).

Characteristics of patients' tumors were evaluated according to their BRCA mutation profiles (Table 1). As regards the histological type of tumors, invasive ductal carcinomas were found in 260 (86\%), invasive lobular in 20 (6.6\%), and other types in $22(7.3 \%)$ patients. Disease stage, tumor histology, mean tumor size, axillary nodal status, perineural invasion, and $\mathrm{Ki}-67$ proliferation index were comparable among BRCA1 mutation carriers, BRCA2 mutation carriers, and non-carriers (Table 1). However, the rate of lymphovascular invasion was significantly higher in BRCA1 mutation carriers $(78 \%)$ than in BRCA2 mutation carriers (54.1\%) and non-carriers (55.3\%) (Table $1)$.

The interrelationship between the estrogen receptor (ER) status, progesterone receptor (PR) status, Her2-neu status, histologic grade, and BRCA mutation profiles of our patients was also evaluated. BRCA1 mutation carriers were more likely to be diagnosed with triplenegative breast cancer $(56.1 \%)$ than non-carriers (32.2\%) and BRCA2 mutation carriers $(29.7 \%)(\mathrm{p}=0.01)$ (Table 1). ER, PR, and HER$2 /$ neu states were comparable between BRCA1/2 mutation carriers and non-carriers. Moreover, $65.9 \%$ of BRCA1 mutation carriers had histologic grade 3 tumor, compared with $32.2 \%$ and $37.8 \%$ in BRCA non-carriers and BRCA2 mutation carriers, respectively (Table 1). The distribution of BRCA1 carriers was also evaluated according to the triple-/non-triple-negative status and histologic grade of the tumor (Table 2). Moreover, $87 \%$ of the triple-negative BRCA1 mutation carriers had histologic grade 3 tumor, compared with $38.9 \%$ of nontriple-negative BRCA1 mutation carriers, and the difference was significant (Table 2).

\section{Discussion and Conclusion}

In this study, we identified the clinical and pathological characteristics of patients based on their BRCA mutation profiles from a cohort of Turkish patients with high-risk breast cancer. We found that more cases of BRCA1-related breast cancers were triple-negative with a higher ratio of histologic grade 3 tumor and lymphovascular invasion than were BRCA-negative and BRCA2-related breast cancers, which are usually associated with a poor prognosis.

BRCA mutation carriers have a very high-risk of breast cancer by age 70 , with incidence of $47 \%-66 \%$ (1). Overall, mutations in these genes are implicated in approximately $15 \%$ of women with familial breast cancer and a similar proportion of all women with incidental ovarian cancers (6). Because women with BRCA mutation-associated breast cancer also have an elevated risk of other malignancies, identifying these mutations is essential for genetic counseling, testing, screening, and prevention strategies (1). However, the prevalence of BRCA1/2 mutation varies based on several factors, including ethnicity, age at diagnosis, sex, tumor histology, and family history $(1,2)$. A few studies from Turkey have reported that the mutation prevalence in patients with high-risk breast carcinoma ranged from $14 \%$ to $19 \%(3,4)$. In our study, the total prevalence rates of BRCA, BRCA1, and BRCA2 mutations were $24 \%, 13.6 \%$, and $12.3 \%$, respectively, among patients with high-risk breast cancer. In a study conducted in Malaysia, patients were grouped according to their personal/family history, and the likelihood of having these mutations was reported highest (60\%) in patients with breast and ovarian cancer, followed by patients with 
Table 1. Clinical and pathological characteristics of patients with breast cancer according to BRCA mutation status.

\begin{tabular}{|c|c|c|c|c|c|}
\hline \multirow{2}{*}{ Characteristics } & & \multirow{2}{*}{$\begin{array}{l}\text { BRCA1 carriers } \\
\qquad \mathbf{n = 4 1}(\%)\end{array}$} & \multirow{2}{*}{$\begin{array}{l}\text { BRCA2 carriers } \\
\qquad n=37(\%)\end{array}$} & \multirow{2}{*}{$\begin{array}{l}\text { BRCA non-carriers } \\
\qquad \mathbf{n = 2 2 7}(\%)\end{array}$} & \multirow{2}{*}{ p-value } \\
\hline & & & & & \\
\hline \multirow{2}{*}{ Age (years) } & $\leq 40$ & $10(24.4)$ & $19(51.4)$ & $105(46.3)$ & \multirow{2}{*}{0.021} \\
\hline & $>40$ & $31(75.6)$ & $18(48.6)$ & $122(53.7)$ & \\
\hline BMI $\left(\mathrm{kg} / \mathrm{m}^{2}\right)$ & & $28.4 \pm 5.5$ & $28.7 \pm 6.8$ & $27.6 \pm 5.0$ & 0.583 \\
\hline \multirow{2}{*}{ Menapousal status } & Premenopausal & $28(68.3)$ & $26(74.3)$ & $151(67.4)$ & \multirow{2}{*}{0.718} \\
\hline & Postmenopausal & $13(31.7)$ & $9(25.7)$ & $73(32.6)$ & \\
\hline Mean tumor size (mm) & & $32.9 \pm 21.3$ & $31.9 \pm 22.1$ & $29.2 \pm 17.2$ & 0.618 \\
\hline Axillary nodal status & Positive & $16(42.1)$ & $11(39.3)$ & $95(49.7)$ & 0.453 \\
\hline Lymphovascular invasion & Positive & $32(78.0)$ & $20(54.1)$ & $125(55.3)$ & 0.022 \\
\hline Perineural invasion & Positive & $28(68.3)$ & $18(48.6)$ & $114(50.7)$ & 0.099 \\
\hline \multirow{3}{*}{ Tumor histology } & IDC & $35(85.4)$ & $34(91.9)$ & $194(85.5)$ & \multirow{3}{*}{0.451} \\
\hline & ILC & $1(2.4)$ & $1(2.7)$ & $18(7.9)$ & \\
\hline & Others & $5(12.2)$ & $2(5.4)$ & $15(6.6)$ & \\
\hline \multirow{3}{*}{ Ki-67(\%) } & $\leq 5 \%$ & $1(2.4)$ & $2(5.4)$ & $20(8.8)$ & \multirow{3}{*}{0.585} \\
\hline & $5 \%-20 \%$ & $12(29.3)$ & $14(37.8)$ & 77 (33.9) & \\
\hline & $>20$ & $28(68.3)$ & $21(56.8)$ & $130(57.3)$ & \\
\hline Triple-negative & & $23(56.1)$ & $11(29.7)$ & $73(32.2)$ & 0.010 \\
\hline ER & Positive & $17(41.5)$ & $23(62.2)$ & $139(61.2)$ & 0.055 \\
\hline PR & Positive & $15(36.6)$ & $19(51.4)$ & $113(49.8)$ & 0.274 \\
\hline Her2/neu & Positive & $3(7.3)$ & $6(16.2)$ & $44(19.4)$ & 0.169 \\
\hline \multirow{3}{*}{ Histologic grade } & 1 & $2(4.9)$ & $0(0.0)$ & $11(4.8)$ & \multirow{3}{*}{$<0.001$} \\
\hline & 2 & $12(29.3)$ & $23(62.2)$ & $143(63)$ & \\
\hline & 3 & $27(65.9)$ & $14(37.8)$ & $73(32.2)$ & \\
\hline \multirow{4}{*}{ Stage } & 1 & $7(17.1)$ & $8(21.6)$ & $39(17.2)$ & \multirow{4}{*}{0.280} \\
\hline & 2 & $22(53.7)$ & $14(37.8)$ & $115(50.7)$ & \\
\hline & 3 & $7(17.1)$ & $12(32.4)$ & $63(27.8)$ & \\
\hline & 4 & 5 (12.2) & $3(8.1)$ & $10(4.4)$ & \\
\hline
\end{tabular}

Significant p-values are shown in bold and italic.

IDC: Invasive ductal carcinoma; ILC: Invasive lobular carcinoma; BMI: Body mass index; ER: Estrogen receptor; PR: Progesterone receptor; n: Number

Table 2. Distribution of BRCA1 mutation carriers according to the receptor status and histologic grade of the tumor

\begin{tabular}{|c|c|c|c|c|c|}
\hline & \multicolumn{4}{|c|}{ BRCA1 mutation carriers } & \multirow{3}{*}{ p-value } \\
\hline & \multicolumn{2}{|c|}{ Triple-negative } & \multicolumn{2}{|c|}{ Non-triple-negative } & \\
\hline & $\mathbf{n}$ & $\%$ & $\mathbf{n}$ & $\%$ & \\
\hline Grade 1 & 1 & 4.3 & 1 & 5.6 & \\
\hline Grade 2 & 2 & 8.7 & 10 & 55.6 & 0.001 \\
\hline Grade 3 & 20 & 87.0 & 7 & 38.9 & \\
\hline
\end{tabular}


breast carcinoma with a family history of ovarian cancer (50\%), similar to our results (7).

In the present study, no significant difference was found between BRCA mutation profile and stage, tumor histology, mean tumor size, axillary nodal status, perineural invasion, and Ki-67 proliferation index. Numerous studies have also reported that tumor size, tumor type, and axillary nodal status were not significantly different between patients according to the BRCA mutation profile, similar to our results (8-11). However, in our study, lymphovascular invasion was more often noted in BRCA1 mutation carriers (78\%) than in BRCA2 mutation carriers (54.1\%) and non-carriers (55.3\%). A study conducted in Turkey, which evaluated the axillary nodal involvement in patients with breast cancer regardless of BRCA mutation status, reported that lymphovascular invasion occurred in $59.2 \%$ of their patients, which was lower than that in BRCA1 mutation carriers in our study (12). Additionally, a few studies have evaluated the incidence of lymphovascular invasion in patients with BRCA-related breast cancer, but did not find a significantly higher incidence of lymphovascular invasion in BRCA1 mutation carriers (8-10, 13-16). In one of these previous studies, which is a hospital-based cohort study analyzing whether tumors of patients with BRCA1-associated breast carcinomas are different from those of patients with breast cancer without BRCA mutation, the lymphovascular invasion was more common $(50 \%)$ in BRCA1 mutation carriers than in noncarriers $(21 \%)$, but the difference did not reach significance (8). In another study, lymphatic invasion was noted in a higher proportion $(52.5 \%$ and $52.4 \%)$ of BRCA1 mutation carriers than of BRCA1 non-carriers $(26.2 \%$ and $35.7 \%)$ with familial and sporadic breast cancer, respectively (16). Interestingly, in a previous study, lymphovascular invasion was more often reported (53\%) in BRCA2 mutation carriers than in BRCA1 mutation carriers (39\%) and noncarriers (48\%) (17).

In some studies that have analyzed the distribution of hormonal status in BRCA mutation carriers, BRCA1 and BRCA2 mutation carriers were grouped together instead of being examined as two different groups, culminating with the inconsistent results $(14,18)$. However, in subsequent studies, when BRCA1 and BRCA2 mutation carriers were grouped separately, BRCA1 mutation carriers were more likely to be diagnosed with triple-negative breast cancer than non-carriers, and pathological characteristics were comparable between BRCA2 mutation carriers and non-carriers, similar to our results $(5,17$, 19). In studies that have analyzed the relationship between BRCA mutation profile and triple-negative tumor pathology, 50\%-88\% of BRCA1 mutation carriers were diagnosed with triple-negative breast cancer against $14.6 \%-34 \%$ in BRCA mutation non-carriers (17, 20-22). Additionally, in a study of a large group of patients with breast cancer, triple-negative breast cancer was diagnosed in $57.1 \%$, $23.3 \%$, and $13.8 \%$ of BRCA1 mutation carriers, BRCA2 mutation carriers, and non-carriers, respectively (5). In our study, the ratio of patients with triple-negative cancer based on the BRCA mutation profiles was consistent with those reported in above-mentioned previous studies $(5,17,20-22)$. In some studies including different ethnic groups, a hormone receptor status was evaluated in BRCA1 mutation carriers, and these patients were more likely to have ERnegative breast cancer $(5,8,9,17,19)$. In our study, the number of ER-negative BRCA1 mutation carriers was higher than those of BRCA2 mutation carriers and non-carriers, but the difference did
Tumors in BRCA1 carriers had a higher histologic and nuclear grades than those in BRCA non-carriers $(5,8,9,17)$. In our study, $65.9 \%$ of BRCA1 mutation carriers had histologic grade 3 tumors compared with $32.3 \%$ and $37.8 \%$ of non-carriers and BRCA2 mutation carriers, respectively. In another study, BRCA1 mutation carriers with triplenegative disease were reported as having higher nuclear grade (grade 3, 93.5\%) than non-triple-negative BRCA1 mutation carriers (grade 3, $75 \%)$ (5). In our study, BRCA1 mutation carriers with triple-negative disease had a higher histologic grade (grade 3, 87\%) than non-triplenegative BRCA1 mutation carriers (grade 3, 38.9\%). The ratio of nontriple-negative BRCA1 mutation carriers with a higher histologic grade was lower $(38.9 \%)$ in our study than in the aforementioned study that evaluated the nuclear grade status (75\%) in these patients (5).

This study has some limitations. First, this had a retrospective design, which may have restricted the retrieval of the data from patient archives. Second, it was conducted in two tertiary care centers in İstanbul. However, no studies have investigated Turkish patients with breast cancer and focused on the clinical and pathological characteristics of these patients based on their BRCA mutation profiles. Therefore, to the best of our knowledge, this study is the first to report this issue that represents the Turkish population in a large series of patients with high-risk breast cancer.

In conclusion, in this study, more patients with BRCA1-related breast cancers had triple-negative disease, poorly differentiated with a high histologic grade, and a higher ratio of lymphovascular invasion than patients with BRCA-negative and BRCA2-related breast cancers. In our clinical practice, all these findings, which are usually associated with a poor prognosis, support that BRCA1-related breast cancers represent a distinct group of patients with unique clinical and pathological features from other patients with breast cancer.

Ethics Committee Approval: The study was conducted in accordance with the Declaration of Helsinki and approved by the Ethics Committee of İstanbul Professor Doctor Cemil Tasçıŏlu City Hospital (approval no: 48670771514.10/210).

Informed Consent: Retrospective study.

Peer-review: Externally peer-reviewed.

\section{Authorship Contributions}

Conception: M.M.A., Ç.G., Ş.S., N.Y., A.S., Se.A., M.G.; Design: M.M.A., Ç.G., Ş.S., S.A., R.Ç., O.C., Ş.C., A.S., Se.A., M.G.; Supervision: Ç.G., S.A., R.Ç., N.Y., O.C., Ş.C., A.S., Se.A., M.G.; Materials: B.E., N.Y., O.C., Se.A., M.G.; Data Collection or Processing: M.M.A., B.E., Ş.C., S.A., R.Ç., N.Y., O.C., Ş.C., Se.A., M.G.; Analysis or Interpretation: M.M.A., Ç.G., B.E., S.A., R.Ç., N.Y., O.C., Ş.C., A.S., Se.A., M.G.; Literature Search: M.M.A., S.A., R.Ç., A.S; Writing: M.M.A., Ç.G., Ş.S., N.Y., O.C., Ş.C., A.S; Critical Review: M.M.A., Ç.G., Ş.S., S.A., R.Ç., N.Y., O.C., Ş.C., A.S., Se.A., M.G.

Conflict of Interest: No conflict of interest was declared by the authors.

Financial Disclosure: The authors declared that this study received no financial support.

\section{References}

1. Bayraktar $S$, Arun B. BRCA mutation genetic testing implications in the United States. Breast 2017; 31: 224-232, (PMID: 27931006) [CrossRef]

2. Bayraktar S, Jackson M, Gutierrez-Barrera AM, Liu D, Meric-Bernstam F, Brandt A, et al. Genotype-phenotype correlations by ethnicity and 


\section{Atcl et al. Breast Cancer Regarding the BRCA Profile}

mutation location in BRCA mutation carriers. Breast J 2015; 21: 260267 (PMID: 25789811) [CrossRef]

3. Cecener G, Egeli U, Tunca B, Erturk E, Ak S, Gokgoz S, et al. BRCA1/2 germline mutations and their clinical importance in Turkish breast cancer patients. Cancer Invest 2014; 32: 375-387. (PMID: 24884828) [CrossRef]

4. Geredeli C, Yasar N, Sakin A. Germline mutations in BRCA1 and BRCA2 in breast cancer patients with high genetic risk in Turkish population. Int J Breast Cancer 2019; 9645147. doi: 10.1155/2019/9645147 (PMID: 30713775) [CrossRef]

5. Atchley DP, Albarracin CT, Lopez A, Valero V, Amos CI, GonzalezAngulo AM, et al. Clinical and pathologic characteristics of patients with BRCA-positive and BRCA-negative breast cancer. J Clin Oncol 2008; 26 : 4282-4288. (PMID: 18779615) [CrossRef]

6. Alsop K, Fereday S, Meldrum C, deFazio A, Emmanuel C, George J, et al. BRCA mutation frequency and patterns of treatment response in BRCA mutation-positive women with ovarian cancer: a report from the Australian Ovarian Cancer Study Group. J Clin Oncol 2012; 30: 26542663. (PMID: 22711857) [CrossRef]

7. Thirthagiri E, Lee SY, Kang P, Lee DS, Toh GT, Selamat S, et al. Evaluation of BRCA1 and BRCA2 mutations and risk-prediction models in a typical Asian country (Malaysia) with a relatively low incidence of breast cancer. Breast Cancer Res 2008; 10: R59. doi: 10.1186/bcr2118 (PMID:18627636) [CrossRef]

8. de Bock GH, Tollenaar RA, Papelard H, Cornelisse CJ, Devilee P, van de Vijver MJ. Clinical and pathological features of BRCA1 associated carcinomas in a hospital-based sample of Dutch breast cancer patients. $\mathrm{Br}$ J Cancer 2001; 85: 1347-1350. (PMID: 11720473) [CrossRef]

9. Quenneville LA, Phillips KA, Ozcelik H, Parkes RK, Knight JA, Goodwin PJ, et al. HER-2/neu status and tumor morphology of invasive breast carcinomas in Ashkenazi women with known BRCA1 mutation status in the Ontario Familial Breast Cancer Registry. Cancer 2002; 95: 20682075. (PMID: 12412159) [CrossRef]

10. Heerma van Voss MR, van der Groep P, Bart J, van der Wall E, van Diest PJ. Lympho-vascular invasion in BRCA related breast cancer compared to sporadic controls. BMC Cancer 2010; 10: 145. (PMID: 20398395) [CrossRef]

11. Robson ME, Chappuis PO, Satagopan J, Wong N, Boyd J, Goffin JR, et al. A combined analysis of outcome following breast cancer: differences in survival based on BRCA1/BRCA2 mutation status and administration of adjuvant treatment. Breast Cancer Res 2004; 6: R8-R17. (PMID: 14680495) [CrossRef]

12. Sakin A, Aldemir MN. Lymph node ratio predicts long-term survival in lymph node-positive breast cancer. Eur J Breast Health 2020; 16: 270275. (PMID: 33062968) [CrossRef]
13. Eisinger F, Nogues C, Guinebretiere JM, Peyrat JP, Bardou VJ, Noguchi T, et al. Novel indications for BRCA1 screening using individual clinical and morphological features. Int J Cancer 1999; 84: 263-267. (PMID: 10371344) [CrossRef]

14. Robson M, Rajan P, Rosen PP, Gilewski T, Hirschaut Y, Pressman P, et al. BRCA associated breast cancer: absence of a characteristic immunophenotype. Cancer Res 1998; 58: 1839-1842. (PMID: 9581822) [CrossRef]

15. Noori SF, Gangi A, Nelson ME, Choi M, Mirzadehgan P, Bonk AK, et al. Comparison of nodal metastasis between BRCA mutation carriers and non-BRCA mutation carriers with breast cancer. Ann Surg Oncol 2014; 21: 3324-3329. (PMID: 25047471) [CrossRef]

16. Feng L, Jin F. Expression and prognostic significance of Fanconi anemia group D2 protein and breast cancer type 1 susceptibility protein in familial and sporadic breast cancer. Oncol Lett 2019; 17: 3687-3700. (PMID: 30881493) [CrossRef]

17. Copson ER, Maishman TC, Tapper WJ, Cutress RI, Greville-Heygate S, Altman DG, et al. Germline BRCA mutation and outcome in youngonset breast cancer (POSH): a prospective cohort study. Lancet Oncol 2018; 19: 169-180. (PMID: 29337092) [CrossRef]

18. Veronesi A, de Giacomi C, Magri MD, Lombardi D, Zanetti M, Scuderi C, et al. Familial breast cancer: characteristics and outcome of BRCA 1-2 positive and negative cases. BMC Cancer 2005; 5: 70. (PMID: 15996267) [CrossRef]

19. Noguchi S, Kasugai T, Miki Y, Fukutomi T, Emi M, Nomizu T. Clinicopathologic analysis of BRCA1- or BRCA2-associated hereditary breast carcinoma in Japanese women. Cancer 1999; 85: 2200-2205. (PMID: 10326698) [CrossRef]

20. Musolino A, Bella MA, Bortesi B, Michiara M, Naldi N, Zanelli P, et al: BRCA mutations, molecular markers, and clinical variables in earlyonset breast cancer: A population-based study. Breast 2007; 16: 280-292. (PMID: 17257844) [CrossRef]

21. Li WF, Hu Z, Rao NY, Song CG, Zhang B, Cao MZ, et al. The prevalence of $\mathrm{BRCA} 1$ and $\mathrm{BRCA} 2$ germline mutations in high-risk breast cancer patients of Chinese Han nationality: two recurrent mutations were identified. Breast Cancer Res Treat 2008; 110: 99-109. (PMID: 17851763) [CrossRef]

22. Byrski T, Gronwald J, Huzarski T, Grzybowska E, Budryk M, Stawicka $\mathrm{M}$, et al. Response to neo-adjuvant chemotherapy in women with BRCA1-positive breast cancers. Breast Cancer Res Treat 2008; 108: 289296. (PMID: 17492376) [CrossRef] 